\title{
Simple Coding for Achieving Mean Square Stability over Bit-rate Limited Channels
}

\author{
Eduardo I. Silva, Milan S. Derpich, Jan Østergaard and Daniel E. Quevedo
}

\begin{abstract}
The problem of characterizing lower bounds on data-rates needed for closed loop stability has been solved in a variety of settings. However, the available results lead to coding schemes which are very complex and, thus, of limited practical interest. In this paper, we show how simple coding systems comprising only LTI filters and memoryless entropy coded dithered scalar quantizers can be used to stabilize strongly stabilizable SISO LTI plant models over error-free bit-rate limited feedback channels. Despite the simplicity of the building blocks employed, we prove that the data-rates incurred do not exceed absolute lower bounds by more than 1.25 bits per sample.
\end{abstract}

\section{INTRODUCTION}

The study of networked control systems (NCSs), i.e., control systems with communication constraints, has emerged as an active research field during the past years (see, e.g., the special issue [1]). Key questions within this framework are related to the way in which network artifacts affect the stability and performance of control loops that employ non-transparent communication channels. Typical channel artifacts include data-rate limits, random delays and data dropouts. A unifying framework for the treatment of the general NCS analysis or design problem does not exist. Nevertheless, there has been significant progress in the study of subproblems. For example, data-rate constraints have been studied in, e.g., [2]-[5]. The issue of data dropouts has been studied in, e.g., [6] and random time delays have been considered in, e.g., [7].

In this paper we focus on feedback loops closed over delay- and error-free bit-rate limited channels. Within this context, a key existing result establishes necessary and sufficient conditions on the channel data-rate that allows one to achieve closed loop stability (in an appropriate sense; see, e.g., [2], [3], [5] and the many references therein). This result is given in terms of a lower bound on the channel datarate (which depends on the unstable plant poles only) over which coding schemes can be constructed so as to achieve stability. These coding schemes are quite complex and are not attractive from a practical point of view. On the other hand, showing that the rate at which a stable control system is transmitting data is always greater than the aforementioned bound (i.e., necessity) is fairly simple (see also [8], [9]), whereas constructing an actual coding scheme that achieves

E.I. Silva, M.S. Derpich and D.E. Quevedo are with the School of Electrical Engineering and Computer Science, The University of Newcastle, NSW 2308, Australia. E-mails: Eduardo.Silva@studentmail.newcastle.edu.au, dquevedo@ieee.org

J. Østergaard is with the Institute of Electronic Systems, Aalborg University, 9220 Aalborg, Denmark. E-mail: janoedieee.org stability at any rate above the absolute limit (i.e., the proof of sufficiency) is much more involved (see [2], [5]). Clearly, the need arises to develop the study of simple stabilizing coding schemes that achieve rates close to the bounds in [2], [3], [5], and of approaches that allow one to exploit the insights obtained when deriving the necessity of the bounds in the construction of coding schemes.

Motivated by the discussion above, this paper shows how simple coding systems comprising only LTI filters and entropy coded dithered quantizers (see, e.g., [10]) can be used to stabilize strongly stabilizable SISO LTI plant models incurring in data rates which exceed the minimal ones established in [2], [3], [5] by no more than 1.25 bits per sample. The excess rate of our simple coding scheme is given by the sum of two terms: a first term due to the divergence of the quantization noise distribution from Gaussianity, and a second term that originates in the inefficiency of the loss-less coder that generates the binary words.

In our results, the coder and decoder architecture plays an essential role, which, given the results in, e.g., [5], is by no means surprising. Our work also sheds light into the reasons why the results in [11] are not always consistent with the lower bound on data-rates for stability studied in [2].

The remainder of this paper is organized as follows: Section II introduces the notation used in the paper. Sections III and IV present the considered setup and coding scheme, respectively. Section V focuses on a simplified setting where Gaussianity assumptions are imposed. Section VI uses the results in Section $\mathrm{V}$ to derive guaranteed upper bounds on the data-rates that allow one to achieve MSS with the proposed coding scheme. Section VII draws conclusions. Due to space constraints, all proofs have been omitted and can be found in [12] or obtained via e-mail from the authors.

\section{NOTATION}

We define $\mathbb{N}_{0} \triangleq\{0,1, \cdots\}$ and $\mathbb{R}_{0}^{+}=\{x \in \mathbb{R}: 0 \leq$ $x<\infty\}$. We use $z$ as both the argument of the z-transform and as the forward shift operator, where the meaning is clear from the context. Given any scalar $x,|x|$ denotes magnitude.

The set of all discrete-time strictly proper real rational transfer functions is denoted by $\mathcal{R}_{s p}$.

Unless otherwise stated, random processes are always scalar and defined for $k \in \mathbb{N}_{0}$, we abbreviate $\{x(k)\}_{k \in \mathbb{N}_{0}}$, $x(k) \in \mathbb{R}$, by $x$, and define $x^{k} \triangleq\{x(0), \cdots, x(k)\}$. $\mathbb{E}\{\cdot\}$ denotes the expectation operator. The variance, at time instant $k$, of a process $x$ is denoted via $\sigma_{x}^{2}(k)$; similarly, if $x$ is a random variable, then $\sigma_{x}^{2}$ denotes its variance. We define $\sigma_{x}^{2} \triangleq \lim _{k \rightarrow \infty} \sigma_{x}^{2}(k)$, provided the limit exists. If $x$ is 


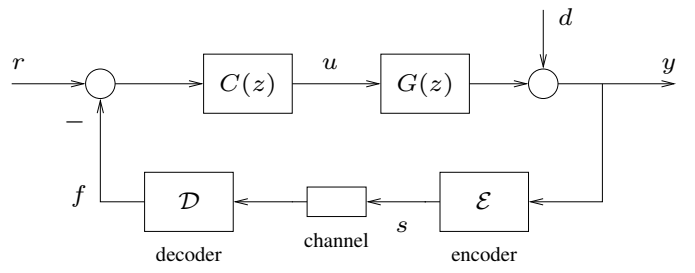

Fig. 1. Considered networked control system (generic coding scheme).

a wide sense stationary (wss) (asymptotically wss) process, then $S_{x}\left(e^{j \omega}\right)$ denotes its (stationary) power spectral density (PSD) and $\Omega_{x}(z)$ denotes any spectral factor of $S_{x}\left(e^{j \omega}\right)$, i.e., $\left|\Omega_{x}\left(e^{j \omega}\right)\right|^{2} \triangleq S_{x}\left(e^{j \omega}\right)$. We say that a random variable is a second order one if and only if it has finite mean and finite and non-zero variance.

If $x, y, z$ are continuous (resp. discrete) random variables, then $h(x)$ (resp. $H(x)$ ) denotes the differential (resp. discrete) entropy of $x ; h(x \mid y)$ (resp. $H(x \mid y)$ ) denotes the conditional differential (discrete) entropy of $x$, given $y ; I(x ; y)$ denotes the mutual information between $x$ and $y ; I(x ; y \mid z)$ denotes the conditional mutual information between $x$ and $y$, given $z$. We recall that if $x$ is Gaussian, then $h(x)=$ $\frac{1}{2} \ln 2 \pi e \sigma_{x}^{2}$. In this paper we use logarithms in base $e$. Thus, information is measured in nats $\left(1\right.$ nat $=(\ln 2)^{-1}$ bits). For details and basic properties of the quantities mentioned above we refer the reader to [13].

\section{Problem Setup}

In this paper we are concerned about the stability of a closed loop system built around a SISO discrete time LTI plant model, which employs a delay- and error-free data-rate limited channel in the feedback path, as shown in Fig. 1. In that figure, $G(z)$ is the plant model, $C(z)$ is an LTI controller, $y$ is the plant output, $u$ is the plant input, $r$ is a reference signal, and $d$ models disturbance signals. In order to make use of the bit-rate limited channel, the feedback path comprises an encoder $\mathcal{E}$ which generates binary words based on information regarding the plant output. These words, which we will denote by $s$, are then sent delay- and errorfree through the channel. At the receiving end, a decoder $\mathcal{D}$ uses the received symbols to generate the signal $f$ that is fed back into the controller.

We will make the following assumptions:

Assumption 1 (Plant model): $G(z) \in \mathcal{R}_{s p}$ is unstable, ${ }^{1}$ has no poles or zeros on the unit circle, is strongly stabilizable ${ }^{2}$ and its initial state is an independent second order random variable.

Assumption 2 (Reference and disturbance): The signals $r$ and $d$ are second order zero-mean wss processes that admit rational PSDs, and that are mutually uncorrelated. Moreover, $S_{d}\left(e^{j \omega}\right)+S_{r}\left(e^{j \omega}\right) \not \equiv 0$.

Assuming that the plant is strictly proper guarantees that the feedback loop in Fig. 1 is well-posed for all causal controllers, decoders and encoders. The assumption regarding

\footnotetext{
${ }^{1}$ Otherwise the problem of stabilization would admit a trivial solution.

${ }^{2}$ I.e., it admits a stable stabilizing controller (see, e.g., [14]).
}

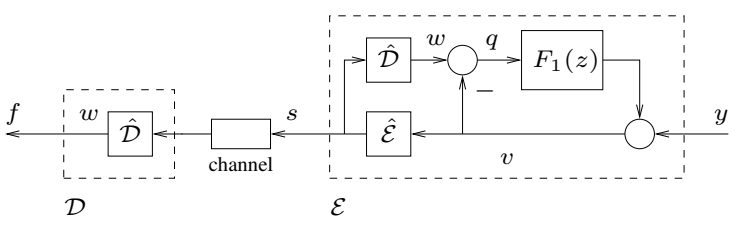

Fig. 2. Proposed coding scheme.

the plant initial state holds for most cases of interest. The remaining assumptions on $G(z)$ are not essential, but we have made them to maintain a straightforward presentation (see [15] for the general case). Assumption 2 is standard except for the fact that we require $r$ or $d$ to be non-zero. We avoid the case $r=d=0$ for brevity.

For future reference, we define $\left\{p_{1}, \cdots, p_{n_{p}}\right\}$ as the set of unstable plant poles, and

$$
\xi_{p}(z) \triangleq \prod_{i=1}^{n_{p}} \frac{1-z p_{i}}{z-p_{i}} .
$$

Throughout this paper we adopt the following notion of stability (see also, e.g., [16]-[18]):

Definition 1 (Mean square stability): Consider a system described by $x(k+1)=f(x(k), w(k), k)$, where $k \in \mathbb{N}_{0}$, $f: \mathbb{R}^{n} \times \mathbb{R}^{m} \times \mathbb{R} \rightarrow \mathbb{R}^{n}, x(k) \in \mathbb{R}^{n}$ is the system state at time instant $k, x(0)=x_{o}$, where $x_{o}$ is a second order random variable, and the input $w$ is a second order wss process independent of $x_{o}{ }^{3}$ We say that the system is mean square stable (MSS) if and only if there exist finite $\mu \in \mathbb{R}^{n}$ and finite $M \in \mathbb{R}^{n \times n}, M \geq 0$, such that

$$
\lim _{k \rightarrow \infty} \mathbb{E}\{x(k)\}=\mu, \quad \lim _{k \rightarrow \infty} \mathbb{E}\left\{x(k) x(k)^{T}\right\}=M,
$$

regardless of the initial state $x_{o}$.

Significant work has been devoted to the study of the MSS of the networked control system in Fig. 1, when arbitrarily complex coding schemes are employed (see, e.g., [2], [5]). However, the study of simple and efficient coding schemes has received much less attention. This is indeed one of the main motivations for the present work.

\section{PRoposed CODING SCHEME}

We seek simple, though effective, coding and decoding schemes. We propose to use the coding scheme shown in Fig. 2. In that figure, $F_{1}(z)$ is an LTI filter, and $\hat{\mathcal{E}}, \hat{\mathcal{D}}$ are (for the moment) abstract systems that are allowed to exploit the history of their inputs to generate their corresponding outputs. More precisely, for every $k \in \mathbb{N}_{0}$,

$$
s(k)=\hat{\mathcal{E}}_{k}\left(v^{k}, s^{k-1}\right), \quad w(k)=\hat{\mathcal{D}}_{k}\left(s^{k}\right),
$$

where $\hat{\mathcal{E}}_{k}$ and $\hat{\mathcal{D}}_{k}$ are (possibly stochastic) mappings that may depend explicitly upon time, and where the range of $\hat{\mathcal{E}}_{k}$ is a collection of prefix-free binary words (see, e.g., [13]). Note that the resulting control loop is well-posed if $F_{1}(z) \in \mathcal{R}_{s p}$.

The coding scheme defined above is a restricted instance of much more general schemes. Indeed, it is a special case of

\footnotetext{
${ }^{3}$ I.e., $w$ is wss, $w(k)$ is a second order random variable for every $k \in \mathbb{N}_{0}$, and $\mathbb{E}\left\{w(i) w(j)^{T}\right\}$ is finite for every $i, j \in \mathbb{N}_{0}$.
} 
a coding scheme that exploits the history of the plant output and of the received symbols in an arbitrary causal fashion (see, e.g., [2], [5]).

We will assume that the following holds:

Assumption 3 (Coding scheme): The coding scheme in Fig. 2 is such that, $\forall k \in \mathbb{N}_{0}$,

(a) knowing $s^{k}$ and $v^{k}$ does not provide more knowledge about $w(k)$ than just knowing $s^{k}$,

(b) the sequence of mappings $\left\{\hat{\mathcal{D}}_{i}\right\}_{i \in\{0, \cdots, k\}}$ is known at the encoder and is such that $s^{k}$ can be recovered exactly from $w^{k}$ (and vice-versa). Also,

(c) $F_{1}(z) \in \mathcal{R}_{s p}$ and the corresponding initial state is an independent second order random variable.

At each time instant $k$ the encoder transmits the symbol $s(k)$ using a word of length $R(k)$ (measured in nats). We will be interested in the average coding rate and, accordingly, we define the average data-rate as

$$
\bar{R} \triangleq \lim _{k \rightarrow \infty} \frac{1}{k} \sum_{i=0}^{k-1} R(i)
$$

provided the limit exists. $\bar{R}$ is the average rate at which the symbols are sent trough the channel and, as such, is a measure of the "information flow" at the physical level.

A key question that arises when considering any coding scheme is how to characterize lower bounds on the achievable average data-rates. To that end we define the following:

Definition 2: Consider two random processes $v$ and $w$. We define (if the defining limit exists) the average directed mutual information between $v$ and $w$ as $^{4}$ (see also [19])

$$
I_{\infty}(v \rightarrow w) \triangleq \lim _{k \rightarrow \infty} \frac{1}{k} \sum_{i=0}^{k-1} I\left(w(i) ; v^{i} \mid w^{i-1}\right) .
$$

We can now characterize lower bounds on the average data-rate for the proposed coding scheme:

Theorem 1 (Lower bounds on data-rates): Consider the networked control architecture in Fig. 1 and the specific coding scheme in Fig. 2, where $\hat{\mathcal{E}}$ and $\hat{\mathcal{D}}$ are as described above. If Assumptions 3(a-b) hold, then $\bar{R} \geq I_{\infty}(v \rightarrow w)$.

Proof: See [12].

Theorem 1 relates a physical quantity, namely average data-rate, to an information theoretic quantity, namely average directed mutual information. It is important to note that a different bound on the average data-rate would have arisen if we had considered $\hat{\mathcal{D}}$ and $\hat{\mathcal{E}}$ with different information available.

Theorem 1 is a key one. However, it is not straightforward to characterize $I_{\infty}(v \rightarrow w)$ unless one makes suitable assumptions on $\hat{\mathcal{E}}$ and $\hat{\mathcal{D}}$ or, equivalently, on the signal

$$
q \triangleq w-v
$$

In the next section we will make some Gaussianity assumptions. Later, in Section VI, we will consider a simple instance of $\hat{\mathcal{E}}$ and $\hat{\mathcal{D}}$ and we will exploit the results for the

\footnotetext{
${ }^{4} I_{\infty}(v \rightarrow w)$ is sometimes called directed mutual information rate.
}

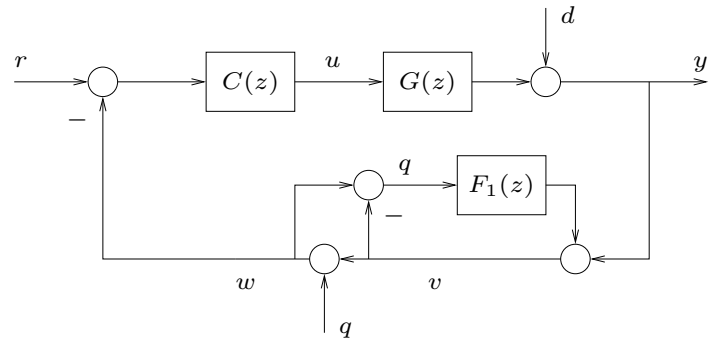

Fig. 3. Model of the networked control system under study.

Gaussian case to provide guaranteed upper bounds on the rates associated to that specific coding scheme, without using the Gaussianity assumptions.

\section{The Gaussian CAse}

As foreshadowed at the end of Section IV, we will assume in this section that the following holds:

Assumption 4 (Gaussianity): The noise $q$ (see (4)) is an independent second order zero-mean i.i.d. Gaussian sequence. The initial states of all LTI filters in Fig. 3 (including plant and controller) are independent second order Gaussian random variables, and $r$ and $d$ are jointly Gaussian processes.

Consistent with the fact that the noise $q$ depends on the way in which $\hat{\mathcal{E}}$ and $\hat{\mathcal{D}}$ are chosen, we will consider the variance of $q$, say $\sigma_{q}^{2}$, as design parameter. Assumption 4, when combined with the networked control system in Fig. 1 and the coding scheme in Fig. 2, yields the linear model in Fig. 3.

We will associate to every pair $\left\{F_{1}(z), C(z)\right\}$ a vector $K(z)$ defined via

$$
K(z) \triangleq\left[F_{1}(z) \quad C(z)\right] .
$$

As long as Assumptions 2 and 4 hold and $q$ has finite variance, the networked control system in Fig. 3 is MSS if and only if $K(z)$ is such that the feedback loop in Fig. 3 is well-posed internally stable in the standard deterministic sense (see, e.g., [20], [21]). Thus, if we define

$$
\begin{aligned}
\mathcal{S} \triangleq\left\{K(z) \in \mathcal{R}_{s p} \times\right. & \mathcal{R}_{p}: \text { the loop in Fig. } 3 \text { is } \\
& \text { internally stable and well-posed }\},
\end{aligned}
$$

then MSS is equivalent to $K(z) \in \mathcal{S}$ and $\sigma_{q}^{2} \in \mathbb{R}_{0}^{+}$(provided Assumptions 2 and 4 hold; see [20]). ${ }^{5}$ It is easy to see that $K(z) \in \mathcal{S}$ if and only if $F_{1}(z)$ is stable, and $C(z)$ is an admissible controller ${ }^{6}$ for $G(z)$ (see, e.g., [21]).

Using Theorem 1 we can now characterize average directed mutual information in the situation under study:

Corollary $1\left(I_{\infty}(v \rightarrow w)\right.$ under Gaussianity assumptions): Consider the feedback loop in Fig. 3 and assume that

\footnotetext{
${ }^{5}$ Consistent with Assumption 3(c), $F_{1}(z)$ is constrained to be strictly proper.

${ }^{6} \mathrm{~A}$ controller is said admissible for a given plant if and only if it is internally stabilizing and defines a well-posed control loop (see [21])
} 
Assumptions 1, 2 and 4 hold. If $K(z) \in \mathcal{S}$ and $\sigma_{q}^{2} \in \mathbb{R}_{0}^{+}$, then

$$
\begin{aligned}
I_{\infty}(v \rightarrow w) & =\frac{1}{4 \pi} \int_{-\pi}^{\pi} \ln \frac{S_{w}\left(e^{j \omega}\right)}{\sigma_{q}^{2}} d \omega \\
& \stackrel{(a)}{\leq} \frac{1}{2} \ln (1+\gamma), \quad \gamma \triangleq \frac{\sigma_{v}^{2}}{\sigma_{q}^{2}},
\end{aligned}
$$

where $S_{w}\left(e^{j \omega}\right)$ is the stationary PSD of $w, \sigma_{v}^{2}$ is the stationary variance of $v$, and $\gamma$ is the stationary coding scheme signal-to-noise ratio. Equality in $(a)$ holds if and only if $S_{w}\left(e^{j \omega}\right) \sigma_{q}^{-2}$ is constant for every $\omega$.

Proof: See [12].

Corollary 1 provides an explicit expression for the average directed mutual information across the coding scheme in the considered situation. Also, it establishes a relationship between the average directed mutual information across the coding scheme and the corresponding signal-to-noise ratio. In particular, it follows from (6) that (provided Assumptions 1,2 and 4 hold) minimizing the coding scheme signal-tonoise ratio amounts to minimizing an upper bound on the average directed mutual information across it. Moreover, if $S_{w}\left(e^{j \omega}\right) \sigma_{q}^{-2}$ is a constant, then we have equality in (6). Hence, under the constraint of having $S_{w}\left(e^{j \omega}\right) \sigma_{q}^{-2}$ constant, minimizing the coding scheme signal-to-noise ratio also minimizes the average directed mutual information across it. We will show below that, in our setting, one can restrict $S_{w}\left(e^{j \omega}\right) \sigma_{q}^{-2}$ to be constant without loss of generality. Towards that goal, we start with the following theorem:

Theorem 2 (Directed mutual information for MSS):

Suppose that Assumptions 1, 2 and 4 holds. Then:

1) There exists $K(z) \in \mathcal{R}_{s p} \times \mathcal{R}_{p}$ such that the feedback loop in Fig. 3 is MSS if and only if

$$
I_{\infty}(v \rightarrow w)>I_{\infty}^{\mathrm{inf}}(v \rightarrow w) \triangleq \sum_{i=1}^{n_{p}} \ln \left|p_{i}\right| .
$$

2) Any $I_{\infty}(v \rightarrow w)>I_{\infty}^{\inf }(v \rightarrow w)$ can be achieved in a MSS loop if one chooses a stable and strictly proper $F_{1}(z)$ such that $1+F_{1}(z)$ is minimum phase (MP), $C(z)$ is any stable admissible controller for $G(z)$, and $\sigma_{q}^{2}$ is made sufficiently large (but finite).

Proof: See [12].

Theorem 2 establishes a bound on the directed mutual information across the considered coding schemes whose satisfaction is necessary and sufficient to achieve MSS. It also provides a characterization of the controller $C(z)$, the LTI filter $F_{1}(z)$, and the noise variance $\sigma_{q}^{2}$, that allow one to achieve any average directed mutual information arbitrarily close to the bound established.

We can use Theorem 1 to immediately infer that, provided the assumptions in Theorem 2 hold,

$$
\bar{R}>\sum_{i=1}^{n_{p}} \ln \left|p_{i}\right|
$$

is a necessary condition that average data-rates need to satisfy in order to guarantee MSS when employing the proposed coding scheme. We note that we have recovered, for the class of considered plant models, the bound on average data-rates for MSS derived in [2]. However, we have not provided a proof of the achievability of this bound. This issue will be explored in Section VI.

We next explore the minimal stationary coding scheme signal-to-noise ratio compatible with MSS. This will enable us to state the main result of this section.

Theorem 3 (Signal-to-noise ratio for MSS): Suppose that Assumptions 1, 2 and 4 hold.

1) There exists $K(z) \in \mathcal{R}_{s p} \times \mathcal{R}_{p}$ such that the feedback loop in Fig. 3 is MSS if and only if the stationary coding scheme signal-to-noise ratio $\gamma$ satisfies

$$
\gamma>\gamma_{\mathrm{inf}} \triangleq\left(\prod_{i=1}^{n_{p}}\left|p_{i}\right|^{2}\right)-1 .
$$

2) Any $\gamma>\gamma_{\text {inf }}$ can be achieved in a MSS loop if one chooses $C(z)$ as any stable admissible controller for $G(z), F_{1}(z)$ is chosen as

$$
F_{1}(z)=\xi_{p}(\infty)\left(\xi_{p}(z) S(z)\right)^{-1}-1,
$$

where $S(z) \triangleq(1+C(z) G(z))^{-1}$, and $\sigma_{q}^{2}$ is made sufficiently large (but finite).

Proof: See [12].

From Theorems 2 and 3 we have the following corollary:

Corollary 2: Consider the feedback loop in Fig. 3 and suppose that Assumptions 1, 2 and 4 hold. A solution to the problem of finding the minimal stationary coding scheme signal-to-noise ratio that guarantees MSS provides a solution to the problem of finding the minimal average directed mutual information across the coding scheme that guarantees MSS.

Proof: See [12].

Corollary 2 is one of the main results in this paper. Indeed, by virtue of Theorem 1, Corollary 2 allows one to conclude that determining the minimal signal-to-noise ratio for MSS provides an immediate lower bound on the average data-rates that allow one to achieve MSS. The key to establishing these facts lies in that we consider a coding architecture with sufficient degrees of freedom. This allows one to make $S_{w}\left(e^{j \omega}\right) \sigma_{q}^{-2}$ constant in the limit as $\sigma_{q}^{2} \rightarrow \infty$, without constraining the minimum achievable directed mutual information across the coding scheme, or the corresponding minimal achievable signal-to-noise ratio (a simple calculation based on the results of Theorem 3 reveals this). We feel that the insights provided by these results are of fundamental importance and, to the best of our knowledge, new.

Remark 1: The work [11] also studies minimal signal-tonoise ratio considerations for stability. However, the authors of [11] constrain themselves to the particular case $F_{1}(z)=0$, which does not, in general, allow one to achieve the absolute minimal coding scheme signal-to-noise ratio identified in Theorem 3. Hence, it is not surprising that the results in [11] do not always suggest bounds on average data-rates that are consistent with the results in [2], where coding schemes that exploit the history of $y$ and $s$ are used. 


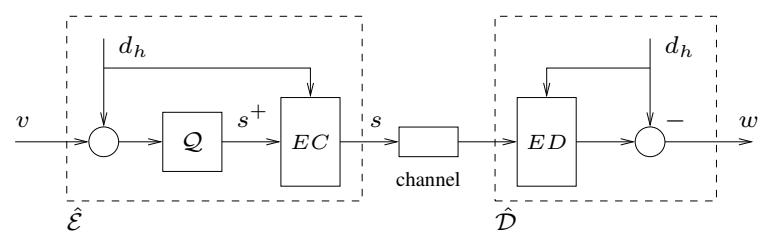

Fig. 4. Entropy coded dithered quantizer.

\section{UpPeR Bounds on ACHIEVABLE DATA-RATES}

Section V has focused on establishing a necessary lower bound on the average data-rate that allows one to guarantee MSS. We have, however, provided no proof of the achievability of this bound. It is well known (see [2], [5]) that there exist coding schemes that allow one to achieve MSS with average data-rates arbitrarily close to the bound identified in Section V. However, since we have chosen a specific coding scheme that is much simpler than those proposed in [2], [5], there exists no guarantee that we will be able to actually achieve the bound. This motivates the study of guaranteed upper bounds on the average data-rate for MSS applicable to the proposed coding scheme. In particular, we will use the results in Section V to study achievable rates, when a memoryless entropy coded dithered quantizer (ECDQ) is used to generate the binary words that are sent over the channel.

Fig. 4 shows the architecture of an ECDQ and its relationship to $\hat{\mathcal{D}}$ and $\hat{\mathcal{E}}$ in Fig. 2 (see, e.g., [10]). The ECDQ is such that

$$
s^{+}(k)=\mathcal{Q}\left(v(k)+d_{h}(k)\right),
$$

where $d_{h}(k)$ is the dither signal which is known at both ends of the channel, and $\mathcal{Q}$ corresponds to a uniform quantizer defined via $\mathcal{Q}(x) \triangleq i \Delta$ for $\left(i-\frac{1}{2}\right) \Delta \leq x \leq\left(i+\frac{1}{2}\right) \Delta$, $i \in \mathbb{Z}$, where $\Delta$ is the quantization step. At each time instant, $s^{+}(k)$ is memory- and loss-lessly coded by the entropy coder EC using explicit information about $d_{h}(k)$ and the corresponding binary word is sent over the channel. Upon reception, the decoder ED, which knows $d_{h}(k)$, decodes the received symbol recovering $s^{+}(k)$. Accordingly, the output $w(k)$ becomes equal to $s^{+}(k)-d_{h}(k)$.

Key properties of ECDQs are the following:

Lemma 1 (ECDQ (see [10])): Consider an ECDQ as described above. If $d_{h}$ is an independent sequence of i.i.d. random variables, uniformly distributed on $\left[-\frac{\Delta}{2}, \frac{\Delta}{2}\right]$, then the noise $q$ defined in (4) is distributed according to the distribution of $-d_{h}$, and the scalar mutual information between $v(k)$ and $w(k)$ satisfies $I(v(k) ; w(k))=H\left(s^{+}(k) \mid d_{h}(k)\right)$.

Remark 2: The proof in [10] assumes no feedback around the ECDQ. If there is strictly causal feedback around it (as in our case), then the same result applies (see detailed proof in [15]).

The results in Lemma 1 state that, if one employs an appropriately dithered ECDQ, then the difference between the input and output of the ECDQ, namely $q$, becomes just an i.i.d. source, uniformly distributed on each quantization interval (as in the classical additive white noise model for quantization; see, e.g., [22], [23]). This means that the use of an ECDQ allows one to achieve a noise $q$ that resembles the noise source considered in Section V (except for having a different distribution). Therefore, it is sensible to expect that use of an appropriately designed ECDQ yields average data-rates "close" to the bounds identified in Section V. We will show below that this is indeed true. We note that, given the fact that the dither signal is known at both ends of the channel, choosing $d_{h}$ as in Lemma 1 implies that the ECDQ satisfies Assumptions 3(a-b) (i.e., an ECDQ is a valid instance of $\hat{\mathcal{D}}$ and $\hat{\mathcal{E}}$ in Fig. 2).

We are now ready to prove the main result of this paper:

Theorem 4 (Achievable rates): Consider the networked control system in Fig. 1 with the coding scheme in Fig. 2, where $\hat{\mathcal{D}}$ and $\hat{\mathcal{E}}$ form an ECDQ as described above. Suppose that the memoryless entropy coder-decoder pair ECED inside the ECDQ uses Huffman coding (see [13]) that Assumptions 1, 2 and 3(c) hold, that the controller initial state is an independent second order random variable, and that the dither signal $d_{h}$ is an independent sequence of i.i.d. random variables, uniformly distributed on $\left[-\frac{\Delta}{2}, \frac{\Delta}{2}\right]$. Then, if one chooses $K(z)$ as in Theorem 3 (Part 2; see (5)) and $\Delta=\sqrt{12 \sigma_{q}^{2}}$, with $\sigma_{q}^{2} \in \mathbb{R}_{0}^{+}$, then the resulting networked control system will be MSS and the corresponding average data-rate will be upper bounded as

$$
\bar{R} \leq \sum_{i=1}^{n_{p}} \ln \left|p_{i}\right|+\frac{1}{2} \ln \left(\frac{2 \pi e}{12}\right)+\ln 2+F(\Delta),
$$

where $F: \mathbb{R}_{0}^{+} \rightarrow \mathbb{R}_{0}^{+}$is a continuous and decreasing function of $\Delta$ such that $\lim _{\Delta \rightarrow \infty} F(\Delta)=0, \lim _{\Delta \rightarrow 0} F(\Delta)=\infty$.

Proof: See [12].

Remark 3 (Bounds are conservative): The upper bound for the average data-rate in Theorem 4 is conservative. Indeed, the rates achieved by Huffman coding are usually closer to the entropy of the source than to the entropy of the source plus $\ln 2$ (see, e.g., [24].). As a consequence, the actual data-rates will be usually close to the expression in Theorem 4 with the term $\ln 2$ omitted.

Theorem 4 is the main result in this paper. It establishes guaranteed upper bounds on the average data-rates that guarantee MSS and that are achievable with the coding scheme in Fig. 2, when $\hat{\mathcal{D}}$ and $\hat{\mathcal{E}}$ form a memoryless ECDQ and when the plant model is strongly stabilizable. If one chooses $K(z)$ as suggested in Part 2 of Theorem 3, and $\Delta$ is chosen sufficiently large (but finite), then one will be able to achieve a rate that is no more than $\frac{1}{2} \ln \left(\frac{2 \pi e}{12}\right)+\ln 2$ nats per sample (i.e., 1.25 bits per sample) away from the absolute bound in [2], [5]. This additional rate is composed by two terms: the first one is due to the divergence of the distribution of quantization noise from Gaussianity, and the second one originates in the inefficiency of the loss-less coding scheme employed to generate the binary words. We feel that this extra rate is a fair price to be paid if one constrain oneself to the conceptually simple coding schemes considered in this paper. 
Remark 4: Note that the results in Theorem 4 rely on the insights developed in Section V. In other words, the insights developed when establishing lower bounds on the average data-rates needed for stabilization were key when constructing an actual coding scheme that achieved a datarate close to that bound. It is also worth noting that the quantization scheme considered in this paper constitutes a well studied building block in the Information Theory literature (see, e.g., [10], [23], [25]). All the above stands in stark contrast to the approach in, e.g., [2].

\section{CONCLUSIONS}

In this paper, we have studied the mean square stabilization of strongly stabilizable SISO LTI plant models controlled over bit-rate limited channels. We have proposed a simple coding scheme which uses only LTI filters and an entropy coded dithered quantizer. Within this setup, we have shown that the excess data-rates, which derive from using this reduced-complexity scheme instead of an arbitrarily complex one, are no larger than 1.25 bits per sample. Extensions to more general cases and to performance related questions can be found in [15].

\section{REFERENCES}

[1] P. Antsaklis and J. Baillieul, "Special issue on technology of networked control systems," Proceedings of the IEEE, vol. 95, no. 1, pp. 5-8, January 2007.

[2] G. Nair and R. Evans, "Stabilizability of stochastic linear systems with finite feedback data rates," SIAM Journal on Control and Optimization, vol. 43, no. 2, pp. 413-436, July 2004.

[3] G. Nair, F. Fagnani, S. Zampieri, and R. Evans, "Feedback control under data rate constraints: An overview," Proceedings of the IEEE, vol. 95 , no. 1 , pp. 108-137, January 2007.

[4] A. Savkin, "Analysis and synthesis of networked control systems: Topological entropy, observability, robustness and optimal control," Automatica, vol. 42, pp. 51-62, 2006.

[5] S. Tatikonda and S. Mitter, "Control under communication constraints," IEEE Transaction on Automatic Control, vol. 49, no. 7, pp. 1056-1068, July 2004.

[6] L. Schenato, B. Sinopoli, M. Franceschetti, K. Poolla, and S. Sastry, "Foundations of control and estimation over lossy networks," Proceedings of the IEEE, vol. 95, no. 1, pp. 163 - 187, January 2007.
[7] F. Lian, J. Moyne, and D. Tilbury, "Modelling and optimal controller design of networked control systems with mutliple delays," International Journal of Control, vol. 76, no. 6, pp. 591-606, 2003.

[8] N. Elia, "When Bode meets Shannon: Control oriented feedback communication schemes," IEEE Transaction on Automatic Control, vol. 49, no. 9, pp. 1477-1488, 2004.

[9] N. Martins and M. Dahleh, "Fundamental limitations of performance in the presence of finite capacity feedback," in Proceedings of the American Control Conference, Portland, USA, 2005.

[10] R. Zamir and M. Feder, "On universal quantization by randomized uniform/lattice quantizers," IEEE Transactions on Information Theory, vol. 38, no. 2, pp. 428-436, March 1992.

[11] J. Braslavsky, R. Middleton, and J. Freudenberg, "Feedback stabilization over signal-to-noise ratio constrained channels," IEEE Transactions on Automatic Control, vol. 52, no. 8, pp. 1391-1403, 2007.

[12] E. Silva, M. Derpich, J. Østergaard, and D. Quevedo, "Simple coding for achieving mean square stability over bit-rate limited channels," School of EE\&CS, The University of Newcastle, Australia, Tech. Rep. (http://altair.elo.utfsm.cl/public_elo), 2008

[13] T. Cover and J. Thomas, Elements of Information Theory, 2nd ed. John Wiley and Sons, Inc., 2006.

[14] J. Doyle, B. Francis, and A. Tannenbaum, Feedback Control Theory. New York: Macmillan Publishing Company, 1992.

[15] E. Silva, "A unified framework for the analysis and design of networked control systems," Ph.D. dissertation, School of Electrical Engineering and Computer Science, The University of Newcastle, Australia, 2008.

[16] N. Elia, "Remote stabilization over fading channels," Systems \& Control Letters, vol. 54, no. 3, pp. 237-249, 2005.

[17] F. Kozin, "A survey of stability of stochastic systems," Automatica, vol. 5, pp. 95-112, 1969.

[18] O. Costa and M. Fragoso, "Stability results for discrete-time linear systems with Markovian jumping parameters," Journal of Mathematical Analysis and Applications, vol. 179, pp. 154-178, 1993.

[19] J. Massey, "Causality, feedback and directed information," in Proceedings of the International Symposium on Information Theory and its Applications., Hawaii, USA, 1990.

[20] T. Söderström, Discrete-time stochastic systems. Prentice Hall, 1994.

[21] K. Zhou, J. Doyle, and K. Glover, Robust and optimal control. Englewood Cliffs, N.J.: Prentice Hall, 1996.

[22] N. Jayant and P. Noll, Digital Coding of Waveforms. Principles and Approaches to Speech and Video. Prentice Hall, 1984.

[23] R. Gray and T. Stockham (Jr.), "Dithered quantizers," IEEE Transactions on Information Theory, vol. 39, no. 3, pp. 805-812, May 1993.

[24] R. M. Gray and D. L. Neuhoff, "Quantization," IEEE Transactions on Information Theory, vol. 44, no. 6, pp. 2325-2383, Oct. 1998.

[25] R. Zamir, Y. Kochman, and M. Feder, "Achieving the Gaussian ratedistortion function by prediction," IEEE Transactions on Information Theory, vol. 54, no. 7, pp. 3354 - 3364, July 2008. 